Pacific Journal of Mathematics

ALGEBRAIC PROPERTIES OF THE COMPOSITION OF
SOLUTIONS OF PARTIAL DIFFERENTIAL EQUATIONS 


\title{
ALGEBRAIC PROPERTIES OF THE COMPOSITION OF SOLUTIONS OF PARTIAL DIFFERNTIAL EQUATIONS
}

\author{
R. SHerman LeHMAN
}

1. Introduction. Let $L u=0$ be a homogeneous linear partial differential equation with constant coefficients in two independent variables $x$ and $y$. In [1] Lewy introduced a class of compositions each of which associates with any two solutions $u$ and $v$ of the differential equation a function $w$ which depends bilinearly on $u$ and $v$. The main result of [1] is that $w$ is a solution of the same differential equation. In this paper we first give a simpler proof of this result and then investigate when the composition is commutative and associative. We prove that for every differential equation at least one of the compositions is commutative. For associativity our results are limited to second order equations. We show that when the differential equation is parabolic, one of the compositions is both commutative and associative. For elliptic and hyperbolic equations none of the compositions are associative, but by a suitable modification an operation which is both commutative and associative is obtained.

2. The compositions. The solutions $u$ and $v$ will be assumed to be defined in a domain $\mathscr{D}$ of the $x, y$-plane with the origin $O$ as an interior point. Let $x_{P}, y_{P}$ and $x_{Q}, y_{Q}$ be the coordinates of the points $P$ and $Q$, respectively; and let $P-Q$ be the point with coordinates $x_{P}-x_{Q}, y_{P}-y_{Q}$. We use $\xi$ and $\eta$ to denote operators which map functions into functions such that

$$
\xi f(P)=\frac{\partial}{\partial x_{P}} f(P), \eta f(P)=\frac{\partial}{\partial y_{P}} f(P) .
$$

Note that this implies that

$$
\begin{aligned}
& \xi f(Q)=\frac{\partial}{\partial x_{Q}} f(Q), \eta f(Q)=\frac{\partial}{\partial y_{Q}} f(Q), \\
& \xi f(P-Q)=\frac{\partial}{\partial x_{P}} f(P-Q)=-\frac{\partial}{\partial x_{Q}} f(P-Q) .
\end{aligned}
$$

All derivatives occurring in formulas will be assumed to be continuous so that the operators $\xi$ and $\eta$ commute. When a path of integration from $O$ to $P$ is considered, it will always be assumed that for each

Received June 27, 1962. This research was supported by the Office of Naval Research under Contract Nonr-222 (62). 
point $Q$ of the path both $Q$ and $P-Q$ are in the domain $\mathscr{D}$.

If $L$ is the operator defined by the equation

$$
L u(P)=\Sigma a_{k} \xi^{k} \eta^{l} u(P)
$$

where the sum is over nonnegative integers $k$ and $l$ with $k+l \leqq \rho$, then by Green's formula if $u$ and $v$ have continuous derivatives of order $\leqq \rho$

$$
\begin{aligned}
& \iint_{\mathscr{G}}[u(P-Q) L v(Q)-v(Q) L u(P-Q)] d x_{Q} d y_{Q} \\
& \quad=\int_{\partial \mathscr{G}} B_{1}(u(P-Q), v(Q)) d x_{Q}+B_{2}(u(P-Q), v(Q)) d y_{Q}
\end{aligned}
$$

where the $B_{i}$ are certain bilinear forms in $u$ and derivatives of order $\leqq \rho-1$ evaluated at $P-Q$ on the one hand and $v$ and derivatives of order $\leqq \rho-1$ evaluated at $Q$ on the other. In general, these bilinear forms are not uniquely determined by $L$.

Using an arbitrary choice of the $B_{i}$ for which (2.3) holds, Lewy defines the composition $u \circ v$ of two solutions $u$ and $v$ of

$$
L u(P)=0
$$

by setting

$$
u \circ v(P)=\int_{o}^{P}\left[B_{1}(u(P-Q), v(Q)) d x_{Q}+B_{2}(u(P-Q), v(Q)) d y_{Q}\right] .
$$

By (2.3) the integral is independent of the path from $O$ to $P$. In this way we obtain, in general, not a single composition but a class of compositions.

Let $\mathscr{D}^{\prime}$ be the domain consisting of all points $P$ in $\mathscr{D}$ which can be reached by paths from $O$ such that for each point $Q$ on the path both $Q$ and $P-Q$ are in $\mathscr{D}$. (In particular, if $\mathscr{D}$ is convex, $\mathscr{D}^{\prime}$ is identical with $\mathscr{D}$.) The following theorem is the main result of [1].

THEOREM 1. If $u$ and $v$ are solutions of (2.4) with continuous derivatives of order $2 \rho-1$ in $\mathscr{D}$ and if $u \circ v$ is an arbitrary composition given by (2.5), then $L(u \circ v)=0$ in the domain $\mathscr{D}^{\prime}$.

3. Proof of Theorem 1. We begin by establishing that if the theorem holds for some one of the compositions given by (2.5) it holds for all of them. If $B_{1}, B_{2}$ are the bilinear forms associated with one composition and $B_{1}^{\prime}, B_{2}^{\prime}$ the forms associated with another composition, then by (2.3) for all sufficiently differentiable functions $u(P-Q)$ and $v(Q)$ (whether solutions of (2.4) or not) 


$$
\left[B_{1}(u, v)-B_{1}^{\prime}(u, v)\right] d x_{Q}+\left[B_{2}(u, v)-B_{2}^{\prime}(u, v)\right] d y_{Q}
$$

must be an exact differential of some function $F$. Let us prove that this function $F$ is a bilinear form with constant coefficients in $u, \xi u$, $\eta u, \cdots$ and $v, \xi v, \eta v, \cdots$. By repeated use of the equation

$$
\begin{aligned}
\xi^{k} \eta^{l} u(P-Q) \xi^{m} \eta^{n} v(Q) & =\frac{\partial}{\partial x_{Q}}\left[\xi^{k} \eta^{l} u(P-Q) \xi^{m-1} \eta^{n} v(Q)\right] \\
& +\xi^{k+1} \eta^{l} u(P-Q) \xi^{m-1} \eta^{n} v(Q)
\end{aligned}
$$

we can remove differentiations of $v$ with respect to $x$ in the expression multiplying $d x_{Q}$ in (3.1). Thus we find (3.1) is equal to

$$
\begin{aligned}
\frac{\partial}{\partial x_{Q}}\left(F_{0}(u, v)\right) d x_{Q} & +\frac{\partial}{\partial y_{Q}}\left(F_{0}(u, v)\right) d y_{Q}+F_{1}(u, v) d x_{Q} \\
& +F_{2}(u, v) d y_{Q}
\end{aligned}
$$

where the $F_{i}$ are forms bilinear in $u, \xi u, \eta u, \cdots$ and $v, \xi v, \eta v, \cdots$ and where all derivatives of $v$ in $F_{1}$ are with respect to $y$. Also, we must have $\left(\partial / \partial y_{Q}\right) F_{1}(u, v)=\left(\partial / \partial x_{Q}\right) F_{2}(u, v)$. If $F_{2}$ did not vanish identically, then $\left(\partial / \partial x_{Q}\right) F_{2}(u, v)$ when written as a form in $u, \xi u, \cdots$ and $v, \xi v, \cdots$ would contain a term in which differentiation of $v$ with respect to $x$ occurs. But, in $\left(\partial / \partial y_{Q}\right) F_{1}(u, v)$ all derivatives of $v$ are with respect to $y$. Consequently $F_{2}$ and $F_{1}$ vanish identically, and $F=F_{0}(u, v)$.

We observe that all derivatives of $u$ and $v$ occurring in $F_{0}$ must be of order $\leqq \rho-2$. The difference between the two compositions $F_{0}(u(O), v(P))-F_{0}(u(P), v(O))$ is a linear combination of $u(P), \xi u(P)$, $\cdots, v(P), \xi v(P), \cdots$ with constant coefficients. Now, if $u$ and $v$ are solutions of (2.4) with continuous derivatives of order $\leqq 2 \rho-2$, then because $L$ has constant coefficients, all derivatives of $u$ and $v$ of order $\leqq \rho-2$ are solutions of (2.4). Thus the difference between the two compositions is a solution.

We now define a particular composition, which we shall call the symmetric composition, and verify that it is a solution of the differential equation. Consider the exterior differential form

$$
\left[u(P-Q) \xi^{k} \eta^{l} v(Q)-v(Q) \xi^{k} \eta^{l} u(P-Q)\right] d x_{Q} d y_{Q} .
$$

Regarding $P$ as fixed and differentiating with respect to $Q$, we find that this form is equal to

$$
\begin{aligned}
d & \left\{\left[\sum_{i=0}^{k-1} \xi^{i} v(Q) \xi^{k-1-i} \eta^{l} u(P-Q)\right] d y_{Q}\right. \\
& \left.-\left[\sum_{j=0}^{l-1} \xi^{k} \eta^{j} v(Q) \eta^{l-1-j} u(P-Q)\right] d x_{Q}\right\}
\end{aligned}
$$

and also is equal to 


$$
\begin{aligned}
d\{[ & {\left[\sum_{i=0}^{k-1} \eta^{l} \xi^{i} v(Q) \xi^{k-1-i} u(P-Q)\right] d y_{Q} } \\
& \left.-\left[\sum_{j=0}^{l-1} \eta^{j} v(Q) \eta^{l-1-j} \xi^{k} u(P-Q)\right] d x_{Q}\right\}
\end{aligned}
$$

With

$$
\begin{aligned}
V_{k l}(u, v) & =\int_{o}^{P}\left(\left[\sum_{i=0}^{k-1} \xi^{i} v(Q) \xi^{k-1-i} \eta^{l} u(P-Q)\right] d y_{Q}\right. \\
& \left.-\left[\sum_{j=0}^{l-1} \xi^{k} \eta^{j} v(Q) \eta^{l-1-j} u(P-Q)\right] d x_{Q}\right) \\
V_{k l}^{*}(u, v) & =\int_{0}^{P}\left(\left[\sum_{i=0}^{k-1} \eta^{l} \xi^{i} v(Q) \xi^{k-1-i} u(P-Q)\right] d y_{Q}\right. \\
& \left.-\left[\sum_{j=0}^{l-1} \eta^{j} v(Q) \eta^{l-1-j} \xi^{k} u(P-Q)\right] d x_{Q}\right)
\end{aligned}
$$

we set

$$
\begin{aligned}
w(P) & =\sum_{k, l} a_{k l} V_{k l}(u, v), \\
w^{*}(P) & =\sum_{k, l} a_{k l} V_{k l}^{*}(u, v) .
\end{aligned}
$$

Both $w(P)$ and $w^{*}(P)$ are compositions of $u$ and $v$ of Lewy's type. The proof of Theorem 1 given in [1] was made by verifying that. $L(w)=0$. It turns out, however, to be more convenient to prove that $L(u \circ v)=0$ where $u \circ v$ is the symmetric composition given by the formula

$$
u \circ v(P)=\frac{1}{2}\left(w(P)+w^{*}(P)\right)
$$

The line integrals $V_{k l}$ and $V_{k l}^{*}$ are, in general, not independent of path. However, in computing the partial derivatives of $u \circ v$ it. is convenient to be able to operate on the terms $V_{k l}$ and $V_{k l}^{*}$ separately. For this reason we extend the operators $\xi$ and $\eta$. If

$$
I=f_{0}(P)+\int_{0}^{P}\left(f_{1}(P, Q) d x_{Q}+f_{2}(P, Q) d y_{Q}\right)
$$

then we set

$$
\begin{aligned}
& \xi I=\frac{\partial f_{0}}{\partial x}(P)+f_{1}(P, P)+\int_{o}^{P}\left(\frac{\partial}{\partial x_{P}} f_{1}(P, Q) d x_{Q}+\frac{\partial}{\partial x_{P}} f_{2}(P, Q) d y_{Q}\right) \\
& \eta I=\frac{\partial f_{0}}{\partial y}(P)+f_{2}(P, P)+\int_{o}^{P}\left(\frac{\partial}{\partial y_{P}} f_{1}(P, Q) d x_{Q}+\frac{\partial}{\partial y_{P}} f_{2}(P, Q) d y_{Q}\right) .
\end{aligned}
$$

The consistency of these definitions follows from the usual rules for differentiating an integral which is independent of the path. We: 
note, however, that some care is required in using these extended operators because the extended operators $\xi$ and $\eta$ may not commute.

We have

$$
\begin{aligned}
& V_{k l}(u, v)=V_{0 l}\left(u, \xi^{k} v\right)+V_{k 0}\left(\eta^{l} u, v\right), \\
& V_{k l}^{*}(u, v)=V_{0 l}\left(\xi^{k} u, v\right)+V_{k 0}\left(u, \eta^{l} v\right) .
\end{aligned}
$$

Setting

$$
S_{k n}(u, v)=\sum_{i=0}^{k-1} \sum_{j=0}^{n-1} \xi^{i} \eta^{j} v(P) \xi^{k-1-i} \eta^{n-1-j} u(O)
$$

and applying (3.6), we obtain

$$
\begin{aligned}
& \xi V_{0 l}(u, v)=V_{0 l}(\xi u, v)-S_{1 l}(u, v) \\
& \eta V_{k 0}(u, v)=V_{k 0}(\eta u, v)+S_{k 1}(u, v) \\
& \xi V_{k 0}(u, v)=V_{k 0}(\xi u, v) \\
& \eta V_{0 l}(u, v)=V_{0 l}(\eta u, v) .
\end{aligned}
$$

Moreover, since

$$
\begin{aligned}
& \xi S_{k n}(u, v)+S_{1 n}\left(\xi^{k} u, v\right)=S_{k+1, n}(u, v), \\
& \eta S_{k n}(u, v)+S_{k 1}\left(\eta^{n} u, v\right)=S_{k, n+1}(u, v),
\end{aligned}
$$

we obtain

$$
\begin{aligned}
& \xi^{m} V_{0 l}(u, v)=V_{0 l}\left(\xi^{m} u, v\right)-S_{m l}(u, v), \\
& \eta^{n} V_{k 0}(u, v)=V_{k 0}\left(\eta^{n} u, v\right)+S_{k n}(u, v), \\
& \xi^{m} V_{k 0}(u, v)=V_{k 0}\left(\xi^{m} u, v\right), \\
& \eta^{n} V_{0 l}(u, v)=V_{0 l}\left(\eta^{n} u, v\right) .
\end{aligned}
$$

Hence by (3.7)

$$
\begin{aligned}
\eta^{n} \xi^{m} V_{k l}(u, v) & =V_{0 l}\left(\xi^{m} \eta^{n} u, \xi^{k} v\right)+V_{k 0}\left(\xi^{m} \eta^{n} \eta^{l} u, v\right) \\
& +S_{k n}\left(\xi^{m} \eta^{l} u, v\right)-S_{m l}\left(u, \xi^{k} \eta^{n} v\right) \\
\xi^{m} \eta^{n} V_{k l}^{*}(u, v) & =V_{0 l}\left(\xi^{m} \eta^{n} \xi^{k} u, v\right)+V_{k 0}\left(\xi^{m} \eta^{n} u, \eta^{l} v\right) \\
& -S_{m l}\left(\xi^{k} \eta^{n} u, v\right)+S_{k n}\left(u, \xi^{m} \eta_{l} v\right) .
\end{aligned}
$$

By (2.2) and (3.4) we have

$$
\begin{aligned}
L w(P) & =\sum_{m, n} a_{m n} \eta^{n} \xi^{m} w(P) \\
& =\sum_{m, n} \sum_{k, l} a_{m n} a_{k l} \eta^{n} \xi^{m} V_{k l}(u, v) .
\end{aligned}
$$

If $L u=0$ and $L v=0$, then

$$
\sum_{k, l} a_{k l}\left[V_{0 l}\left(L u, \xi^{k} v\right)+V_{k 0}\left(L \eta^{l} u, v\right)\right]=0 .
$$


Therefore,

$$
L w(P)=\sum_{m, n} \sum_{k, l} a_{m n} a_{k l}\left[S_{k n}\left(\xi^{m} \eta^{l} u, v\right)-S_{m l}\left(u, \xi^{k} \eta^{n} v\right)\right] .
$$

Similarly

$$
\begin{aligned}
L w^{*}(P) & =\sum_{m, n} \sum_{k, l} a_{m n} a_{k l} \xi^{m} \eta^{n} V_{k l}^{*}(u, v) \\
& =\sum_{m, n} \sum_{k, l} a_{m n} a_{k l}\left[-S_{m l}\left(\xi^{k} \eta^{n} u, v\right)+S_{k n}\left(u, \xi^{m} \eta^{l} v\right)\right] .
\end{aligned}
$$

The value of this sum is unchanged if we interchange $k$ and $m$ and interchange $l$ and $n$ throughout. Doing this, we obtain

$$
L w^{*}(P)=-L w(P) .
$$

Thus for the symmetric composition

$$
L(u \circ v)=\frac{1}{2}\left(L w+L w^{*}\right)=0 ;
$$

and, as we have seen, this implies that $L(u \circ v)=0$ for any of the compositions. This completes the proof of Theorem 1 .

If we assume that there is a straight line segment joining $O$ to $P$ which lies entirely in $\mathscr{D}$, then the change of variable $Q^{\prime}=P$ $-Q$ takes the line segment from $O$ to $P$ into the segment from $P$ to $O$. If this path of integration is used, it is easily verified that

$$
V_{k l}(v, u)=V_{k l}^{*}(u, v)
$$

and hence for the symmetric composition $u \circ v(P)=v \circ u(P)$, i.e., the symmetric composition is commutative.

4. A covariant composition. In this section we introduced for operators of all orders a special composition of Lewy's type which we shall call the covariant composition. For operators of order $\leqq 2$ it coincides with the symmetric composition of $\S 3$.

If $u$ is a solution of the differential equation

$$
L u=\Sigma a_{k l} \frac{\partial^{k+l}}{\partial x^{k} \partial y^{l}} u=0
$$

and the linear transformation

$$
\begin{aligned}
& x^{\prime}=\alpha x+\beta y \\
& y^{\prime}=\gamma x+\delta y, \quad \Delta=\alpha \delta-\beta \gamma \neq 0
\end{aligned}
$$

is made, then $u$ considered as a function of $x^{\prime}$ and $y^{\prime}$ satisfies a new differential equation of the same order 


$$
L^{\prime} u=\Sigma a_{k l}^{\prime} \frac{\partial^{k+l}}{\partial x^{\prime k} \partial y^{\prime l}} u=0
$$

For the transformed operator $L^{\prime}$ we can then obtain the Lewy compositions. We now show how to single out a composition such that if $\Delta=1$ the same function $w=u \circ v$ is obtained whether the composition considered is with respect to $L$ or with respect to $L^{\prime}$.

We begin by introducing certain exterior differential forms $\Omega_{n j}$ which depend bilinearly on $u$ and $v$. Let $A$ and $B$ be arbitrary real constants (indeterminates). Then $\Omega_{n j}$ is defined by equating coefficients in the equation

$$
\begin{aligned}
& \sum_{j=0}^{n-1}(A \xi+B \eta)^{j} v(Q)(A \xi+B \eta)^{n-1-j} u(P-Q)\left(A d y_{Q}-B d x_{Q}\right) \\
& \quad=\sum_{j=0}^{n}\left(\begin{array}{c}
n \\
j
\end{array}\right) A^{j} B^{n-j} \Omega_{n j}(u, v) .
\end{aligned}
$$

We define the covariant composition of two solutions $u$ and $v$ of (4.1) by the equation

$$
u \circ v(P)=\int_{o}^{P} \Sigma a_{k l} \Omega_{k+l, k}(u(P-Q), v(Q)) .
$$

Before stating our main result for the covariant composition, let us verify that it is indeed a composition of the type considered by Lewy. Regarding $P$ as fixed and differentiating with respect to $Q$, we obtain

$$
\begin{aligned}
d & \left\{\sum_{j=0}^{n}\left(\begin{array}{c}
n \\
j
\end{array}\right) A^{j} B^{n-j} \Omega_{n j}\right\}=\sum_{j=0}^{n-1}\left\{(A \xi+B \eta)^{j+1} v(Q)(A \xi+B \eta)^{n-1-j} u(P-Q)\right. \\
& \left.-(A \xi+B \eta)^{j} v(Q)(A \xi+B \eta)^{n-j} u(P-Q)\right\} d x_{Q} d y_{Q} \\
& =\left\{(A \xi+B \eta)^{n} v(Q) u(P-Q)-v(Q)(A \xi+B \eta)^{n} u(P-Q)\right\} d x_{Q} d y_{Q} .
\end{aligned}
$$

Hence

$$
d \Omega_{n j}=\left\{\xi^{j} \eta^{n-j} v(Q) u(P-Q)-v(Q) \xi^{j} \eta^{n-\jmath} u(P-Q)\right\} d x_{Q} d y_{Q},
$$

and consequently (4.5) defines a composition.

THEOREM 2. If the linear change of variables (4.2) is made, then the covariant composition with respect to the transformed operator $L^{\prime}$ is equal to 4 times the covariant composition with respect to $L$.

Proof. Starting with $u \circ v(P)$ as given by (4.5) we make the change of variables (4.2) and express $u \circ v(P)$ in terms of the new variables $x^{\prime}$ and $y^{\prime}$. With $\xi^{\prime}=\partial / \partial x^{\prime}$ and $\eta^{\prime}=\partial / \partial y^{\prime}$ we have 


$$
\begin{aligned}
\xi & =\alpha \xi^{\prime}+\gamma \eta^{\prime}, & d y & =\frac{1}{\Delta}\left(\alpha d y^{\prime}-\gamma d x^{\prime}\right), \\
\eta & =\beta \xi^{\prime}+\delta \eta^{\prime}, & -d x & =\frac{1}{\Delta}\left(\beta d y^{\prime}-\delta d x^{\prime}\right) .
\end{aligned}
$$

We define $\Omega_{n j}^{\prime}(u, v)$ by the following equation which corresponds to (4.4):

$$
\begin{aligned}
& \sum_{j=0}^{n-1}\left(A \xi^{\prime}+B \eta^{\prime}\right)^{j} v(Q)\left(A \xi^{\prime}+B \eta^{\prime}\right)^{n-1-j} u(P-Q)\left(A d y_{Q}^{\prime}-B d x_{Q}^{\prime}\right) \\
& \quad=\sum_{j=0}^{n}\left(\begin{array}{c}
n \\
j
\end{array}\right) A^{j} B^{n-j} \Omega_{n j}^{\prime} .
\end{aligned}
$$

We now express the left hand side of (4.4) in terms of $\xi^{\prime}, \eta^{\prime}, d x_{Q}^{\prime}$, $d y_{Q}^{\prime}$. Since

$$
\begin{aligned}
& A \xi+B \eta=(A \alpha+B \beta) \xi^{\prime}+(A \gamma+B \delta) \eta^{\prime} \\
& A d y_{Q}-B d x_{Q}=\frac{1}{\Delta}\left\{(A \alpha+B \beta) d y_{Q}^{\prime}-(A \gamma+B \delta) d x_{Q}^{\prime}\right\},
\end{aligned}
$$

the left hand side of (4.4) is equal to $1 / \Delta$ times the expression obtained by replacing $A$ by $A \alpha+B \beta, B$ by $A \gamma+B \delta, \xi$ by $\xi^{\prime}, \eta$ by $\eta^{\prime}, d x_{Q}$ by $d x_{Q}^{\prime}, d y_{Q}$ by $d y_{Q}^{\prime}$. Therefore

$$
\sum_{j=0}^{n}\left(\begin{array}{c}
n \\
j
\end{array}\right) A^{j} B^{n-j} \Omega_{n j}=\frac{1}{\Delta} \sum_{j=0}^{n}\left(\begin{array}{c}
n \\
j
\end{array}\right)(A \alpha+B \beta)^{j}(A \gamma+B \delta)^{n-j} \Omega_{n j}^{\prime} .
$$

Next we introduce a linear operator $T$ which takes differential forms into differential forms and satisfies the equation

$$
T \Omega_{n j}^{\prime}=\Omega_{n, j+1}^{\prime} \quad(j=0,1,2, \cdots, n-1) .
$$

From (4.9) we obtain

$$
\begin{aligned}
\sum_{j=0}^{n}\left(\begin{array}{c}
n \\
j
\end{array}\right) A^{j} B^{n-j} \Omega_{n j} & =\frac{1}{\Delta} \sum_{j=0}^{n}\left(\begin{array}{c}
n \\
j
\end{array}\right)(A \alpha T+B \beta T)^{j}(A \gamma+B \delta)^{n-j} \Omega_{n_{0}}^{\prime} \\
& =\frac{1}{\Delta}(A \alpha T+B \beta T+A \gamma+B \delta)^{n} \Omega_{n 0}^{\prime} \\
& =\frac{1}{\Delta} \sum_{j=0}^{n}\left(\begin{array}{c}
n \\
j
\end{array}\right) A^{j} B^{n-j}(\alpha T+\gamma)^{j}(\beta T+\delta)^{n-j} \Omega_{n 0}^{\prime} .
\end{aligned}
$$

Therefore

$$
\Omega_{n j}=\frac{1}{\Delta}(\alpha T+\gamma)^{j}(\beta T+\delta)^{n-j} \Omega_{n 0}^{\prime} .
$$

The covariant composition of $u$ and $v$ with respect to the operator $L^{\prime}$ is given by the integral 


$$
\int_{o}^{P} \Sigma a_{k l}^{\prime} \Omega_{k+l, k}^{\prime}(u(P-Q), v(Q)) .
$$

By (4.7) a term $a_{j, n-j} \xi^{n-j}$ in the operator $L$ transforms into

$$
a_{j, n-j}\left(\alpha \xi^{\prime}+\gamma \eta^{\prime}\right)^{j}\left(\beta \xi^{\prime}+\delta \eta^{\prime}\right)^{n-j} .
$$

Hence

$$
\Sigma a_{k l}^{\prime} \Omega_{k+l, k}^{\prime}=\sum_{\substack{0 \leq n \leq \rho \\ 0 \leqq j \leqq n}} a_{j, n-j}\left\{(\alpha T+\gamma)^{j}(\beta T+\delta)^{n-j} \Omega_{n 0}^{\prime}\right\} .
$$

Comparing (4.13) with (4.11), we see that the composition with respect to $L^{\prime}$ is $\Delta$ times the composition with respect to $L$; this completes the proof of the theorem.

REMARK. If the straight line segment from $O$ to $P$ lies in the domain $\mathscr{D}$, then one can easily show that $u \circ v(P)=v \circ u(P)$, i.e. that the covariant composition is commutative. The proof is similar to that given in $\S 3$ for the commutativity of the symmetric composition.

5. Associativity. In this section we consider an arbitrary secondorder operator with constant coefficients,

$$
\begin{aligned}
L u=a_{20} \frac{\partial^{2} u}{\partial x^{2}} & +2 a_{11} \frac{\partial^{2} u}{\partial x \partial y}+a_{02} \frac{\partial^{2} u}{\partial y^{2}}+a_{10} \frac{\partial u}{\partial x} \\
& +a_{01} \frac{\partial u}{\partial y}+a_{00} .
\end{aligned}
$$

Throughout, the composition considered will be the covariant composition of $\S 4$; and the path of integration will be the straight line segment from $O$ to $P$. As we have seen, the covariant composition is commutative. We now investigate when it is associative.

THEOREM 3. For the second-order operator (5.1) and the covariant composition

$$
u \circ(v \circ w)-(u \circ v) \circ w=D v(O)(u(O) w(P)-u(P) w(O))
$$

where

$$
D=a_{11}^{2}-a_{20} a_{02} .
$$

REMARK. From the theorem it follows that the covariant composition is associative if and only if the operator $L$ is parabolic. Note, however, that if the convariant composition is restricted to the subset of solutions of $L u=0$ which vanish at $O$, then it is always associative, 
Proof. It is convenient to define two operations ${ }_{x}^{*}$ and $\underset{y}{*}$ by the equations

$$
\begin{aligned}
& u \underset{y}{*} v=\int_{o}^{P} u(P-Q) v(Q) d y_{Q} . \\
& u \underset{x}{*} v=-\int_{o}^{P} u(P-Q) v(Q) d x_{Q} .
\end{aligned}
$$

where the integrals are taken along the straight line segment from $O$ to $P$. It is well known that these convolution operations are commutative and associative, i.e.,

$$
\begin{array}{ll}
u \underset{x}{*} v=v \underset{x}{*} u, & u \underset{y}{*} v=v \underset{y}{*} u, \\
u \underset{x}{*}(v \underset{x}{*} w)=(u \underset{x}{*} v) \underset{x}{*} w, & u \underset{y}{*}(v \underset{x}{*} w)=(u \underset{y}{*} v) \underset{x}{*} w, \\
u \underset{x}{*}(v \underset{y}{*} w)=(u \underset{y}{*} v), & u \underset{y}{*}(v \underset{y}{*} w)=(u \underset{y}{*} v) .
\end{array}
$$

By a linear transformation of the independent variables the operator $L$ can be brought into the canonical form

$$
\frac{\partial^{2}}{\partial x^{2}}+\varepsilon \frac{\partial^{2}}{\partial y^{2}}+a \frac{\partial}{\partial x}+b \frac{\partial}{\partial y}+c
$$

where $\varepsilon=1$ for elliptic operators, $\varepsilon=0$ for parabolic operators, and $\varepsilon=-1$ for hyperbolic operators. In the following computations we shall assume that $L$ has this form and that $L u=0, L v=0$, and $L w=0$. We have

$$
\begin{aligned}
& u \circ v=v{ }_{y}^{*} \xi u+\xi v \underset{y}{*} u+\varepsilon v * \eta u+\varepsilon \eta v_{x}^{*} u+a v_{y}^{*} u+b v * u, \\
& v \circ w=\xi w * v+w{ }_{y}^{*} \xi v+\varepsilon \eta w{ }_{x}^{*} v+\varepsilon w_{x}^{*} \eta v+a w \underset{y}{*} v+b w \underset{x}{*} v, \\
& \xi(v \circ w)=\xi v \circ w-\varepsilon \eta w(P) v(O)-\varepsilon w(P) \eta v(O)-b w(P) v(O), \\
& \eta(v \circ w)=\eta v \circ w+\xi w(P) v(O)+w(P) \xi v(O)+a w(P) v(O) .
\end{aligned}
$$

Consequently, $u \circ(v \circ w)$ is equal to

$$
\begin{gathered}
\Phi(u, v, w)-\varepsilon v(O) \eta w \underset{y}{*} u-\varepsilon \eta v(O) w \underset{y}{*} u-b v(O) w \underset{y}{*} u+\varepsilon v(O) \xi w \underset{x}{*} u \\
+\varepsilon \xi v(O) w \underset{x}{*} u+a v(O) w \underset{x}{*} u
\end{gathered}
$$

where $\Phi(u, v, w)$ is equal to the sum of the following 36 terms: 


\begin{tabular}{|c|c|c|c|c|}
\hline$\xi w \underset{y}{*} v \underset{y}{*} \xi u$ & $w \underset{y}{*} \xi v \underset{y}{*} \xi u$ & $\varepsilon \eta w \underset{x}{*} v \underset{y}{*} \xi u$ & & \\
\hline$\xi w \underset{y}{*} \xi v \underset{y}{*} u$ & $w \underset{y}{*} \xi^{2} v \underset{y}{*} u$ & $\varepsilon \eta w{ }_{x}^{*} \xi v{ }_{y}^{*} u$ & & \\
\hline$\varepsilon \xi w_{y}^{*} v \underset{x}{*} \eta u$ & $\varepsilon w \underset{y}{*} \xi v \underset{x}{*} \eta u$ & $\varepsilon^{2} \eta w \underset{x}{*} v \underset{x}{*} \eta u$ & & \\
\hline$\varepsilon \xi w \underset{y}{*} \eta v \underset{x}{*} u$ & $\varepsilon w \underset{y}{*} \xi \eta v \underset{x}{*} u$ & $\varepsilon^{2} \eta w \underset{x}{*} \eta v \underset{x}{*} u$ & & \\
\hline$a \xi w \underset{y}{*} v \underset{y}{*} u$ & $a w \underset{y}{*} \xi v \underset{y}{*} u$ & $\operatorname{ca\eta } w \underset{x}{*} v \underset{y}{*} u$ & & \\
\hline$b \xi w \underset{y}{*} v \underset{x}{*} u$ & $b w \underset{y}{*} \xi v \underset{x}{*} u$ & $\varepsilon b \eta w \underset{x}{*} v \underset{x}{*} u$ & & \\
\hline & & $\varepsilon w \underset{x}{*} \eta v \underset{y}{*} \xi u$ & $a w \underset{y}{*} v \underset{y}{*} \xi u$ & $b w \underset{x}{*} v \underset{y}{*} \xi u$ \\
\hline & & $\varepsilon w \underset{x}{*} \xi \eta v \underset{y}{*} u$ & $a w \underset{y}{*} \xi v \underset{y}{*} u$ & $b w \underset{x}{*} \xi v \underset{y}{*} u$ \\
\hline & & $\varepsilon^{2} w \underset{x}{*} \eta v \underset{x}{*} \eta u$ & $\varepsilon a w \underset{y}{*} v \underset{x}{*} \eta u$ & $\varepsilon b w \underset{x}{*} v \underset{x}{*} \eta u$ \\
\hline & & $\varepsilon^{2} w \underset{x}{*} \eta^{2} v \underset{x}{*} u$ & $\varepsilon a w \underset{y}{*} \eta v \underset{x}{*} u$ & $\varepsilon b w \underset{x}{*} \eta v \underset{x}{*} u$ \\
\hline & & $\varepsilon a w \underset{x}{*} \eta v \underset{y}{*} u$ & $a^{2} w \underset{y}{*} v \underset{y}{*} u$ & $a b w \underset{x}{*} v_{y}^{*} u$ \\
\hline & & $\varepsilon b w \underset{x}{*} \eta v \underset{x}{*} u$ & $a b w \underset{y}{*} v \underset{x}{*} u$ & $b^{2} w \underset{x}{*} v \underset{x}{*} u$. \\
\hline
\end{tabular}

If these 36 terms are arranged as a $6 \times 6$ matrix with the first six lines above as the first three columns of the matrix and the last six lines as the last three columns, then terms on the main diagonal are unchanged if $u$ and $w$ are interchanged, while a term off the diagonal is changed into the one symmetric with respect to the diagonal. Hence $\Phi(u, v, w)=\Phi(w, v, u)$. From this it follows easily that

$$
\begin{aligned}
u \circ(v \circ w)-(u \circ v) \circ w= & \varepsilon v(O)\{\eta u \underset{y}{*} w-\xi u * w-\eta w \underset{x}{*} u+\xi w \underset{x}{*} u\} \\
= & \varepsilon v(O)\left\{\int_{o}^{P} w(P-Q) d u(Q)\right. \\
& \left.-\int_{O}^{P} u(P-Q) d w(Q)\right\} \\
= & \varepsilon v(O)\{w(O) u(P)-w(P) u(O)\} .
\end{aligned}
$$

This proves the theorem if $L$ is in the canonical form (5.5). The general case is then easily obtained by using Theorem 2 .

Although for $D \neq 0$ the covariant composition is not associative, there is a modification of this operation which is both commutative and associative. Using o to denote the covariant composition, we let

$$
u \cdot v=u \circ v+c_{1} u(P) v(O)+c_{2} u(O) v(P) .
$$

where $c_{1}$ and $c_{2}$ are constants.

THEOREM 4. The operation - defined by (5.6) is commutative if and only if $c_{1}=c_{2}$. It is associative if and only if $c_{1}=c_{2}$ and $c_{1}^{2}=D$.

Proof. The first statement is obvious. We have 


$$
v \cdot w=v \circ w+c_{1} v(P) w(O)+c_{2} v(O) w(P),
$$

and hence, using the fact that $v \circ w(O)=0$,

$$
\begin{aligned}
& u \cdot(v \cdot w)=u \circ(v \circ w)+c_{1} w(O) u \circ v+c_{2} v(O) u \circ w \\
& \quad+c_{1}\left(c_{1}+c_{2}\right) u(P) v(O) w(O)+c_{2} u(O) v \circ w \\
& \quad+c_{1} c_{2} u(O) v(P) w(O)+c_{2}^{2} u(O) v(O) w(P), \\
& (u \cdot v) \cdot w=(u \circ v) \circ w+c_{1} v(O) u \circ w+c_{2} u(O) v \circ w \\
& \quad+c_{1} w(O) u \circ v+c_{1}^{2} u(P) v(O) w(O) \\
& \quad+c_{1} c_{2} u(O) v(P) w(O)+c_{2}\left(c_{1}+c_{2}\right) u(O) v(O) w(P) .
\end{aligned}
$$

Using Theorem 3 we obtain

$$
\begin{gathered}
u \cdot(v \cdot w)-(u \cdot v) \cdot w=\left(c_{2}-c_{1}\right) v(O) u \circ w \\
\quad+\left(D-c_{1} c_{2}\right) v(O)[u(O) w(P)-u(P) w(O)]
\end{gathered}
$$

from which the second statement of the theorem follows immediately.

Theorem 4 can be used to answer the question of which compositions for second order operators are commutative and associative. The operation $\cdot$ is a composition if and only if $c_{1}=-c_{2}$. In $\S 3$ it was shown that for second order operators an arbitrary composition has the form

$$
u \circ v+c(u(O) v(P)-u(P) v(O))
$$

where $u \circ v$ is the convariant composition. Thus by Theorem 4 the covariant composition is the only compostion which is commutative. Also, it follows from Theorem 4 that a composition is associative if and only if $D=0$ and $c=0$. Thus for parabolic operators only the covariant composition is associative, while for elliptic and hyperbolic operators none of the compositions are associative.

It is interesting to note that for real hyperbolic equations the two modifications of the composition which are commutative and associative, when applied to two real solutions yield real solutions, but for real elliptic equations no such modification exists. In the case of elliptic equations one must consider complex-valued solutions to obtain an associative operation.

\section{BIBLIOGRAPHY}

1. Hans Lewy, Composition of solutions of linear partial differential equations in two independent variables, J. Math. and Mech., 8 (1959), 185-192.

UNIVERSITY OF CALIFORNIA, BERKELEY 


\section{PACIFIC JOURNAL OF MATHEMATICS}

\section{EDITORS}

\section{RalPh S. Phillips}

Stanford University

Stanford, California

M. G. Arsove

University of Washington

Seattle 5, Washington
J. Dugundji

University of Southern California Los Angeles 7, California

Lowell J. Paige

University of California

Los Angeles 24, California

\section{ASSOCIATE EDITORS}
E. F. BECKENBACH
D. DERRY
H. L. ROYDEN
E. G. STRAUS
T. M. CHERRY
M. OHTSUKA
E. SPANIER
F. WOLF

\section{SUPPORTING INSTITUTIONS}

\author{
UNIVERSITY OF BRITISH COLUMBIA \\ CALIFORNIA INSTITUTE OF TECHNOLOGY \\ UNIVERSITY OF CALIFORNIA \\ MONTANA STATE UNIVERSITY \\ UNIVERSITY OF NEVADA \\ NEW MEXICO STATE UNIVERSITY \\ OREGON STATE UNIVERSITY \\ UNIVERSITY OF OREGON \\ OSAKA UNIVERSITY \\ UNIVERSITY OF SOUTHERN CALIFORNIA
}

STANFORD UNIVERSITY

UNIVERSITY OF TOKYO

UNIVERSITY OF UTAH

WASHINGTON STATE UNIVERSITY

UNIVERSITY OF WASHINGTON

AMERICAN MATHEMATICAL SOCIETY

CALIFORNIA RESEARCH CORPORATION SPACE TECHNOLOGY LABORATORIES

NAVAL ORDNANCE TEST STATION

Printed in Japan by International Academic Printing Co., Ltd., Tokyo Japan 


\section{Pacific Journal of Mathematics}

\section{Vol. 13, No. 1 \\ March, 1963}

Frantz Woodrow Ashley, Jr., A cone of super-(L) functions............. 1

Earl Robert Berkson, Some metrics on the subspaces of a Banach space....

Felix Earl Browder and Walter Strauss, Scattering for non-linear wave

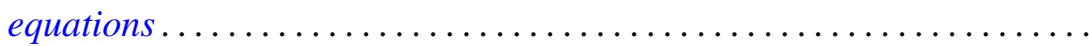

Edmond Darrell Cashwell and C. J. Everett, Formal power series ..........

Frank Sydney Cater, Continuous linear functionals on certain topological

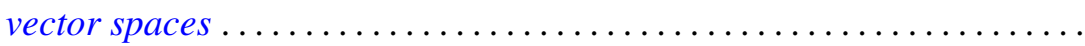

John Douglas Dixon, General group extensions ....................

Robert Pertsch Gilbert, On harmonic functions of four variables with

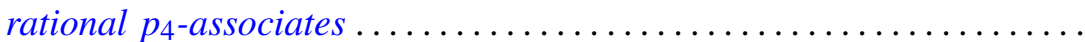

Irving Leonard Glicksberg, On convex hulls of translates ..............

Simon Hellerstein, On a class of meromorphic functions with deficient zeros

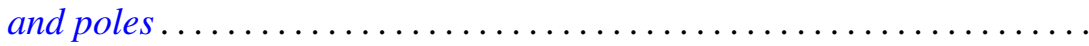

Donald William Kahn, Secondary cohomology operations which extend the

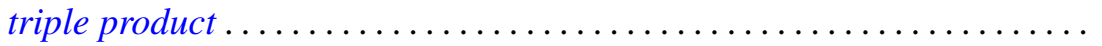

G. K. Leaf, A spectral theory for a class of linear operators .............

R. Sherman Lehman, Algebraic properties of the composition of solutions of partial differential equations ........................... 157

Joseph Lehner, On the generation of discontinuous groups ............. 169

S. P. Lloyd, On certain projections in spaces of continuous functions ...... 171 Fumi-Yuki Maeda, Generalized spectral operators on locally convex spaces ..................................

Donald Vern Meyer, $E^{3}$ modulo a 3-cell

William H. Mills, An application of linear programming to permutation groups.

Richard Scott Pierce, Centers of purity in abelian groups

Christian Pommerenke, On meromorphic starlike functions ...

Zalman Rubinstein, Analytic methods in the study of zeros of

polynomials...

B. N. Sahney, On the Nörlund summability of Fourier series

Tôru Saitô, Regular elements in an ordered semigroup . .

Lee Meyers Sonneborn, Level sets on spheres...........

Charles Andrew Swanson, Asymptotic estimates for limit point

problems .

Lucien Waelbroeck, On the analytic spectrum of Arens . .

Alvin (Murray) White, Singularities of a harmonic function of three

variables given by its series development .............

Kōichi Yamamoto, Decomposition fields of difference sets ...

Chung-Tao Yang, On the action of $\mathrm{SO}(3)$ on a cohomology manifold... 